\title{
Plasma Model for Charging Damage
}

Michael C. Vella, ${ }^{a}$ Wes Lukaszek, ${ }^{b}$ Michael I. Current, ${ }^{c}$ and Nicholas H. Tripsas ${ }^{d}$

\author{
aElectro-Graph, Inc., Carlsbad, CA 92009 \\ bWafer Charging Monitors, Woodside, CA 94062 \\ cApplied Materials, Implant Division, Santa Clara, CA 95054 \\ dAdvanced Micro Devices, Sunnyvale, CA 94088
}

July 1994

This work was partially supported by the Director, Office of Energy Research, Office of Fusion Energy, Development and Technology Division, of the U.S. Department of Energy under Contract No. DE-ACO3$76 \mathrm{SF} 00098$. 


\section{DISCLAIMER}

This report was prepared as an account of work sponsored by an agency of the United States Government. Neither the United States Government nor any agency thereof, nor any of their employees, make any warranty, express or implied, or assumes any legal liability or responsibility for the accuracy, completeness, or usefulness of any information, apparatus, product, or process disclosed, or represents that its use would not infringe privately owned rights. Reference herein to any specific commercial product, process, or service by trade name, trademark, manufacturer, or otherwise does not necessarily constitute or imply its endorsement, recommendation, or favoring by the United States Government or any agency thereof. The views and opinions of authors expressed herein do not necessarily state or reflect those of the United States Government or any agency thereof. 


\section{DISCLAIMER}

Portions of this document may be illegible in electronic image products. Images are produced from the best available original document. 
IIT/94, Catania, Italy, June 13-17

\title{
PLASMA MODEL FOR CHARGING DAMAGE
}

\author{
Michael C. Vella, ${ }^{a}{ }^{*}$ Wes Lukaszek, ${ }^{b}$ Michael I. Current, ${ }^{c}$ and Nicholas H. Tripsas ${ }^{d}$ \\ a Electro-Graph, Inc., Carlsbad, CA 92009 USA \\ b Wafer Charging Monitors, Woodside, CA 94062 USA \\ c Applied Materials, Implant Division, Santa Clara, CA 95054 USA \\ d Advanced Micro Devices, Sunnyvale, CA 94088 USA
}

The mechanism responsible for charging damage is treated [2] as beam/plasma driven differences in local floating potentials on the process surface. A cold plasma flood is shown to limit these potential differences. Beam/plasma $\mathrm{J}-\mathrm{V}$ characteristics obtained with CHARM2 in a high current implanter are fit with the theory. With flood OFF, the fit corresponds to plasma buildup over the target surface.

\section{Introduction}

Charging damage during ion implantation has been a subject of interest for many years [1], and is becoming an issue for plasma tools. The mechanism responsible for damage to integrated circuit device insulators is described [2] as plasma driven differences in local floating potentials on the process surface. During ion implantation, the secondary charged particle coefficient is shown to be the dominant term driving surface potential differences, but can be compensated by sufficient density of thermal plasma electrons near the surface. The plasma model is fit to beam/plasma J-V characteristics obtained with CHARM2.

\section{Background: Plasma Properties}

The space potential in a neutralized ion beam has previously been solved explicitly as a Poisson problem [3]. Potentials in the beam were found to be of the order of tens of volts. The requirements for charged particles to be a plasma [4] are: (1) Scale size much larger than the Debye length, $L_{D}$; and (2) Large number of particles in a Debye sphere. The Debye length can be written, $L_{D}=743\left(T_{e} / n_{e}\right)^{1 / 2}$, where $L_{D}$ is in $\mathrm{cm}, T_{e}$ in eV and $n_{e}$ in $\mathrm{cm}^{-3}$. The particle ion beam density can be written, $n_{i b}=j_{i b} /\left(e Z_{i b} v_{i b}\right)=1.43 E 8\left(j_{i b} / Z_{i b}\right)\left(A_{b} / E_{b}\right)$, ${ }^{1 / 2}$ where $j_{i b}$ is 
the current density in $\mathrm{mA} / \mathrm{cm}^{2} ; Z_{i b}$, the charge state; $A_{b}$, the mass in amu; and $E_{b}$, the energy in $\mathrm{keV}$. If the electron density is approximately equal to the beam ion density, it is trivial to show that a neutralized implanter ion beam easily satisfies the plasma criterion, even for a beam current density $<1 \mathrm{~mA} / \mathrm{cm}^{2}$.

Electron neutralization of an ion beam is difficult to avoid in the absence of applied acceleration fields. Even small deviations from neutralization can cause space charge blowup or dosing non-uniformity. In a plasma, the condition of nearly zero net charge density is called quasineutrality, i.e., $\sum_{\beta} n_{e} \approx \sum_{\alpha} n_{i, \alpha}$, where the sums are over all charge species. Besides space charge blowup, the high potentials associated with an un-neutralized ion beam can cause physical damage to the target by unipolar arcing. Unipolar arcing is more extreme than the kind of oxide wear relevant to processing today, and is not treated here.

The mechanism which produces quasineutrality is the ambipolar plasma potential. Ambipolarity results from the relatively high mobility of electrons compared with ions. This causes most plasmas to have a positive space potential, $\varnothing_{p}$, called the (ambipolar) plasma potential. The plasma potential is usually a multiple of $T_{e}$, and has the effect of electrostatically confining the lowest energy electrons. High mobility also means that electrons also have a high self-collision rate and rapid thermalization. In the absence of a magnetic field, electrons tend to a Boltzman distribution, $n_{e}(x)=n_{o} \operatorname{Exp}\left(V-e \varnothing_{p}(x) / T_{e}\right)$, where $V(x)-e \varnothing_{p}$ represents the difference between the local floating potential and plasma potential. Electron thermal conductivity is so high that $T_{e}$ is usually isotropic.

\section{Charging Model}

A dc plasma model is appropriate for charging phenomena because floating potentials are ultimately arbitrated by electrons, and electron-electron phenomena occur on the shortest time scale, $\leq 100 \mathrm{nsec}$. The net current density on a process surface is written:

$$
j_{\text {net }}=j_{i b}(1+\gamma)+j_{i p}+j_{e t h},
$$

where $j_{i b}(1+\gamma)$ denotes the combination of ion beam current density and total secondary charged particle coefficient; $j_{i p}$, the low energy ion current density; and, $j_{e, t h}$, the thermal electron term. In a plasma tool, the ion beam term would be replaced by an energetic electron current density. Secondary charged particle processes, represented by $\gamma$, are determined by beam and target properties. For structures smaller than a Debye length, the trajectories of low 
energy charged particles near the surface may be affected by local electric fields, but this is ignored here.

Assuming quasi-neutrality, the electron density can be written $n_{e} \approx n_{i b}+n_{i p}$. The local electrical thermal electron current density is [5], $j_{e t h}=e v_{e t h} n_{e} / 4=0.5 e n_{e}\left(2 T_{e} / \pi m_{e}\right)^{1 / 2}$, where $n_{e}$ represents the electron density and $v_{e}$, the thermal electron speed. Since absolute densities at the target surface may be difficult to measure, it is useful to formulate in terms of relative densities. The plasma ion current density at the surface is approximately, $j_{i p}=0.5 e Z_{i} n_{i p}\left(T_{e} / A m_{p}\right)^{1 / 2}$, where $m_{p}$ is the proton mass and $A$ is in amu. Using quasineutrality, the thermal electron current density can be rewritten,

$j_{e t h}(x)=42.8(2 A / \pi)^{1 / 2} j_{i p}\left(1+n_{i b} / n_{i p}\right) \operatorname{Exp}\left[e\left(V-\varnothing_{p}\right) / T_{e}\right]$

The factor 42.8 comes from the square root of the proton-electron mass ratio, and $Z_{i}=1$. Combining Eqs. (1) and (2), the full expression for the net current density becomes,

$$
j_{\text {net }}=j_{i b}(1+\gamma)+j_{i p}\left[1-\left(1+n_{i b} / n_{i p}\right) 34.2 A^{1 / 2} \operatorname{Exp}\left(\left(V-\varnothing_{p}\right) / T_{e}\right)\right]
$$

Where appropriate, each term could become a sum over sub-species.

Since device damage is caused by current flow resulting from potential differences, it is useful to invert Eq.(3). A conducting probe will float (i.e., draw zero net current) at a potential difference with respect to the plasma given by,

$$
\left(V-\varnothing_{p}\right) / T_{e}=\operatorname{Ln}\left[\left(1+(1+\gamma) j_{i b} / j_{i p}\right) /\left(1+n_{i b} / n_{i p}\right)\right]-\operatorname{Ln}\left[34.2 A^{1 / 2}\right] .
$$

Without beam, this gives the usual plasma result, $\left(V-\varnothing_{p}\right) / T_{e} \approx-\operatorname{Ln}\left[34.2 A^{1 / 2}\right] . . \quad$ Eq. [4] also gives the usual implant result that the local floating potential on a process surface which passes through a positive ion beam with surrounding plasma can alternately change sign.

\section{Charging Control: Cold Plasma Flood}

A mechanism to control charging damage is embedded in Eq. [4]. If a separtely generated plasma floods the system, so that $(1+\gamma) j_{i b} / j_{i p}<<1$, the floating potential would everywhere be dominated by the plasma, and several vendors now offer plasma floods. The 
role of plasma ions is to maintain (via quasi-neutrality) a thermal electron population at the process surface, which compensates local potential differences.

With a plasma flood, $V-\varnothing_{p}$ could be minimized by using light plasma ions (which implies increased gas flow), or, by reducing the electron temperature, which is called a cold plasma flood. A magnetic filter [6] can be used to obtain a cold electron temperature plasma, or, the filter effect can be incorporated into an extended magnetic field [7].

\section{Fit to CHARM Data}

Data from the CHARM2 charging diagnostic [8] is used to illustrate the utility of the plasma model. Each $150 \mathrm{~mm}$ wafer has 188 die, and each die has a set of bipolar EEPROM based sensors. CHARM2 can be viewed as an array of device size plasma probes, and used to characterize the charged particle processing environment on a device scale across the entire process surface. The EEPROM's are preset to record peak induced voltages and currents during a process step. The data used here are from a series of resistors on each die which are tied between a $0.24 \mathrm{~mm}^{2}$ conductive pad on the surface and the substrate. The resistors are tapped in lengths which correspond to multiples of two in resistance, up to 1024 times the lowest resistance. The voltage across each resistor is tied to the control gate of a preset EEPROM. Those resistors connected to negatively preset EPROM's (to read positive current) were individually calibrated on an automated Reedholm tester, which was also used for data analysis. For this experiment, the detectable range of current densities was $100 \mathrm{~mA} / \mathrm{cm}^{2}$ to 40 $\mu \mathrm{A} / \mathrm{cm}^{2}$, with \pm 6 Volt threshold voltage. Data shown here were taken on an Applied Materials 9500 implanter at AMD, using a $16 \mathrm{~mA}, 60 \mathrm{keV}$ As beam with plasma flood OFF. With plasma flood $O N$, the data fell into $\pm 6 \mathrm{~V}$ deadband of this set of EEPROM's.

Die near the edge of the wafer corresponded to the leading and trailing edges of the beam. These recorded significantly higher voltages than die in the wafer center. Following CHARM2 numbering, the following die locations were analyzed in detail: D18; D40; D67; D122; D149; and D171. These diagonally cross the wafer in the fast scan beam direction. Data from the negative current sensors were below threshold. Data near +6 Volts were too close to threshold, and usually discarded. The electrical characteristics of the beam/plasma are summarized by J-V plots at these six locations, shown in Fig. 1. Data from the resistor set each die map the beam/plasma J-V characteristic at that location. This corresponds to a plasma probe response in the region between the ion saturation current and the floating potential, with the caveat that the CHARM2 sign convention is opposite the usual for a plasma probe. Here, positive current 
corresponds to a positive charge flowing from the conductor to the substrate. J-V characteristics at D18 and D171 suggest possible damage to $110 \AA$ oxides [9].

Interpretation of CHARM2 data requires care, because the potential difference between the wafer substrate and plasma is uncertain. The wafer floats relative to local electrical ground, but cannot be viewed as a probe, because it forms a significant part of the plasma boundary.

The potential at which the net current goes to zero is the floating potential of the probe relative to the substrate. From Fig. 1, the floating potential depends on location. From Eq. [4], this suggests a large variation in beam and plasma density ratio across the wafer. The highest floating potential is at D18, which corresponds to the center of the beam leading edge. When the beam first reaches D18, which is first exposed to the beam leading edge when most of the beam is over the beam dump. So, this location probably corresponds to minimum plasma generation by beam ionization of atoms from the surface. CHARM2 is a passive diagnostic which provides no data from the electron saturation region, so the electron temperature cannot be determined experimentally. Reasonable fits to the data at D18 were obtained by assuming a bi-Maxwellian electron population (usual in beam-plasma systems) with temperatures of $5 \mathrm{eV}$ and $1 \mathrm{eV}$, each weighted at 50\%. Other parameters used to fit D18 with Eq.(3) were: $j_{i b} / j_{i p}=11.8 ; n_{i b} / n_{i p}=0.3$; and $(1+\gamma) j_{i b}=4.1$. Absent information about the mass of plasma ions, $A_{p}$ was set to 1 , and all plasma densities are stated in hydrogen equivalent units. For these parameters, the rhs of Eq.(4) is approximately zero, which means $V(D 18) \approx$ $\varnothing_{p}=18.9 \mathrm{~V}$. The J-V data for the remaining five die could then be fit by varying only $j_{i b} / j_{i p}$ (or, proportionally, $n_{i b} / n_{i p}$ ), as illustrated in Fig. 2a,b. This assumes the electron temperature is essentially isotropic and constant. Fig. 3 shows a plot of the values of $j_{i b} / j_{i p}$ and $n_{i b} / n_{i p}$ used for the fits, $0.4<j_{i b} / j_{i p}<11.8$, which corresponds to a factor of thirty variation in $j_{i b} / j_{i p}$. This is too large to be attributed to the beam alone. So, the interpretation is that significant plasma buildup occurs as the beam moves from wafer edge to center. No other reasonable parametric variation was found which fit the data at all sites.

\section{Acknowledgments}

*On leave from the Lawrence Berkeley Laboratory, MS 47-112, Berkeley, California 94720, USA. This work partially supported by the Director, Office of Energy Research, Office of Fusion Energy, Development and Technology Division, of the U.S. Department of Energy under Contract No. DE-AC03-76SF00098.

\section{References}

[1] For a recent review see, M.E. Mack, "Handbook of Ion Implantation Technology," J.F. Ziegler (Ed.), North-Holland (1992). 
[2] M.C. Vella, Lawrence Berkeley Laboratory Report, LBL-34970 (June 1993).

[3] A.J.T. Holmes, Phys. Rev. 19(1) (1979), 389-407.

[4] F.F. Chen, "Introduction to Plasma Physics," Plenum Press.

[5] F.F. Chen, "Plasma Diagnostic Techniques," R.H. Huddlestone and S.L. Leonard, Ed., p.113-199, Academic Press (1965).

[6] K.W. Ehlers and K.N. Leung, Rev. Sci. Instrum., 52(10) (1981) 1452.

[7] M.C. Vella, W.S. Cooper, P.A. Pincosy, R.V. Pyle, P.D. Weber, and R.P. Wells, Rev. Sci. Instrum. 59 (1988) 2357.

[8] W. Lukaszek, W. Dixon, M. Vella, C. Messick, S. Reno, and J. Shideler, International Reliability Physics Symposium, San Jose, CA, April 11-14, 1994.

[9] M.I. Current, these proceedings. 
Fig. 1. Current density plotted vs potential for the reistor sets at six die sites going diagnollay across the wafer: D18; D40; D67; D122; D149; and D171.

Fig. 2. Calculated one parameter $\left(j_{i b} / j_{i p}\right)$ fits to the $\mathrm{J}-\mathrm{V}$ data are plotted at sites: $(2 \mathrm{a}) \mathrm{D} 18$, D40, and D67; and (2b) D122, D149, and D171.

Fig. 3. The values of $j_{i b} / j_{i p}$ used to fit the model to the data plotted vs die number (right axis); $n_{i p} / n_{i b}$ is plotted on the left axis. 


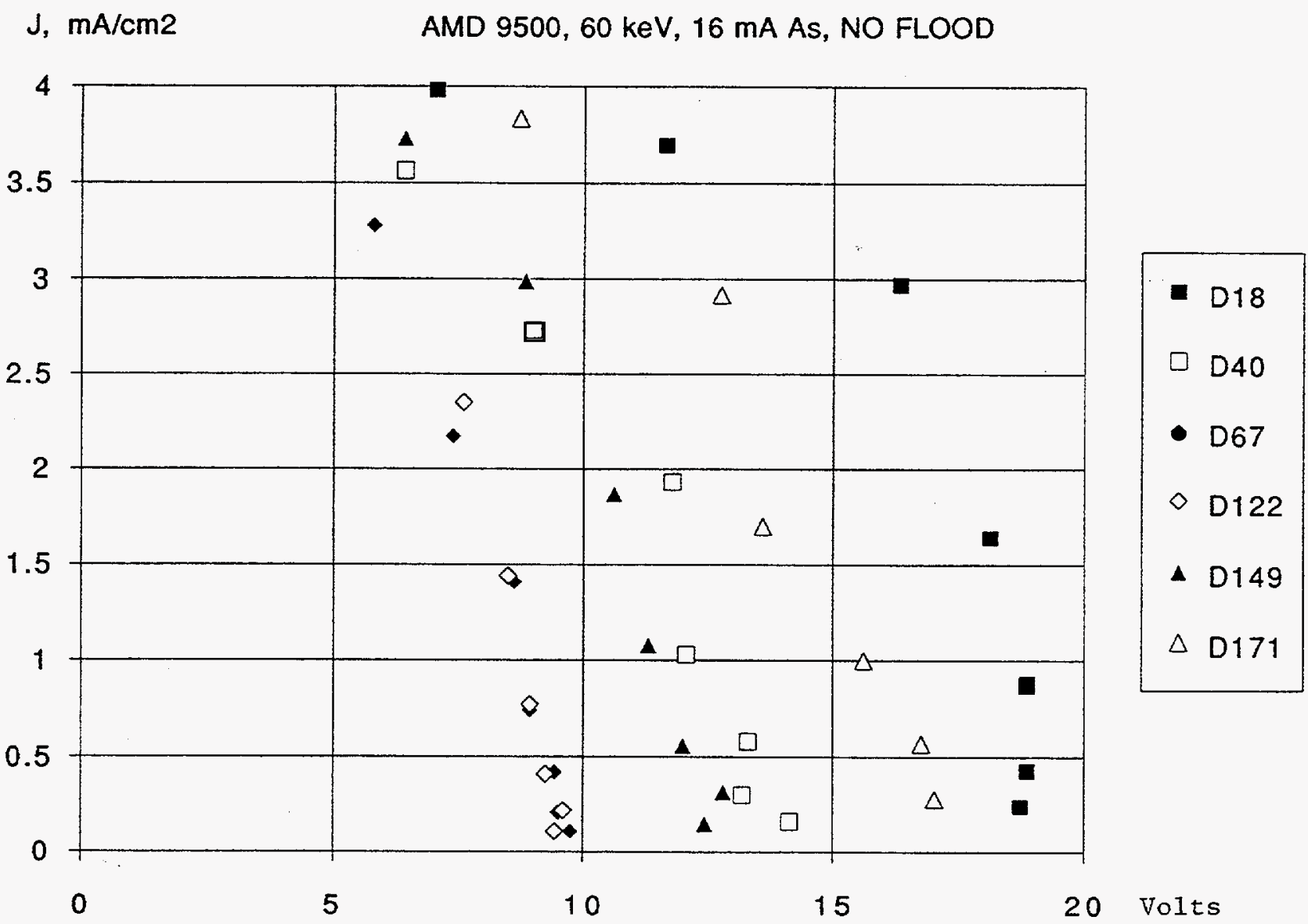

Fig. 1. Current density plotted vs potential for the resistor sets at six die sites going diagonally across the wafer: D18; D40; D67; D122; D149; and D171. 


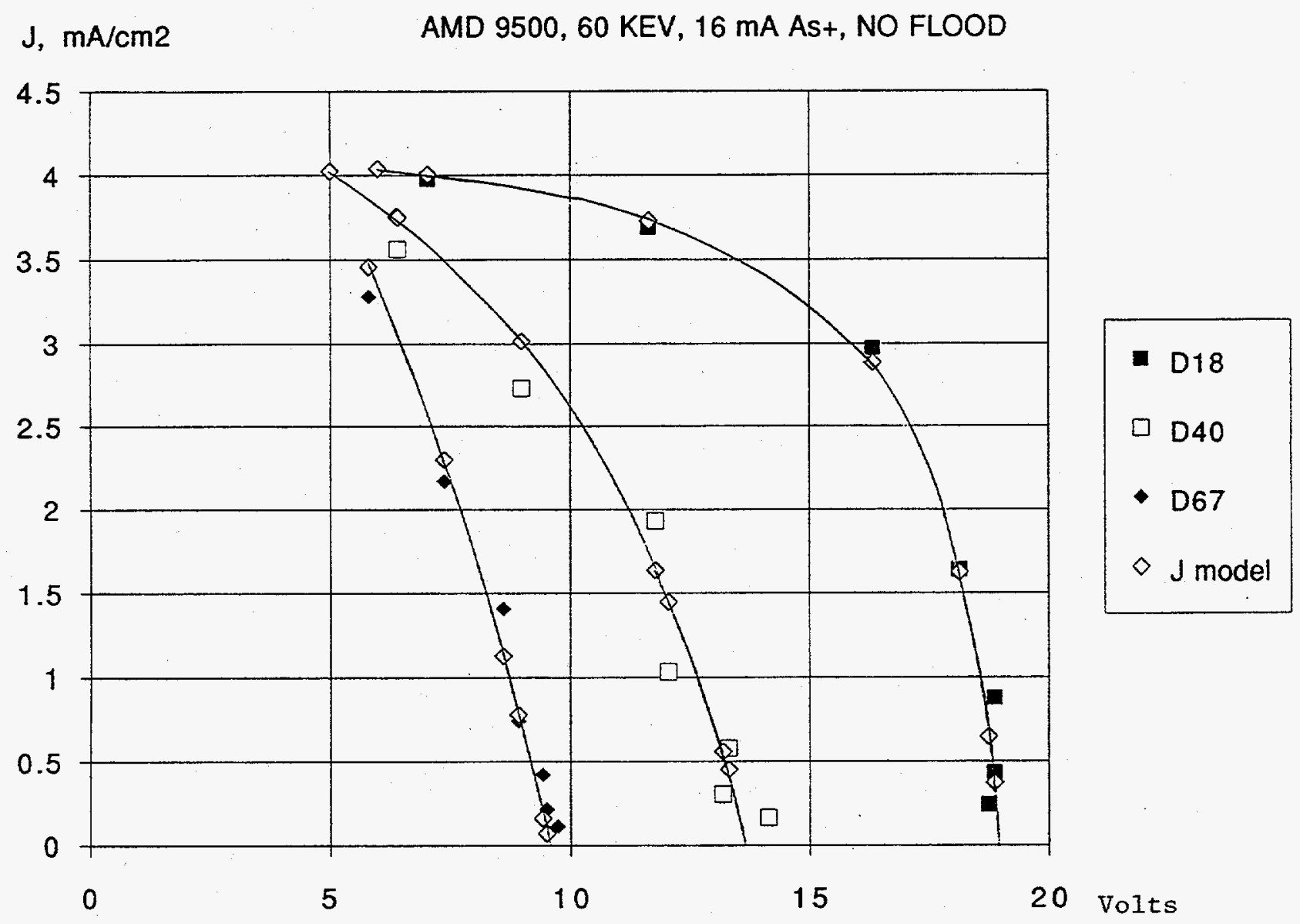

Fig. 2a. Calculated one parameter $\left(j_{i b} / j_{i p}\right)$ fits to the $J-V$ data plotted at sites: D18; D40; and D67. 

$\mathrm{J}, \mathrm{mA} / \mathrm{cm} 2$
AMD 9500, $60 \mathrm{KEV}, 16 \mathrm{~mA}$ As+, NO FLOOD

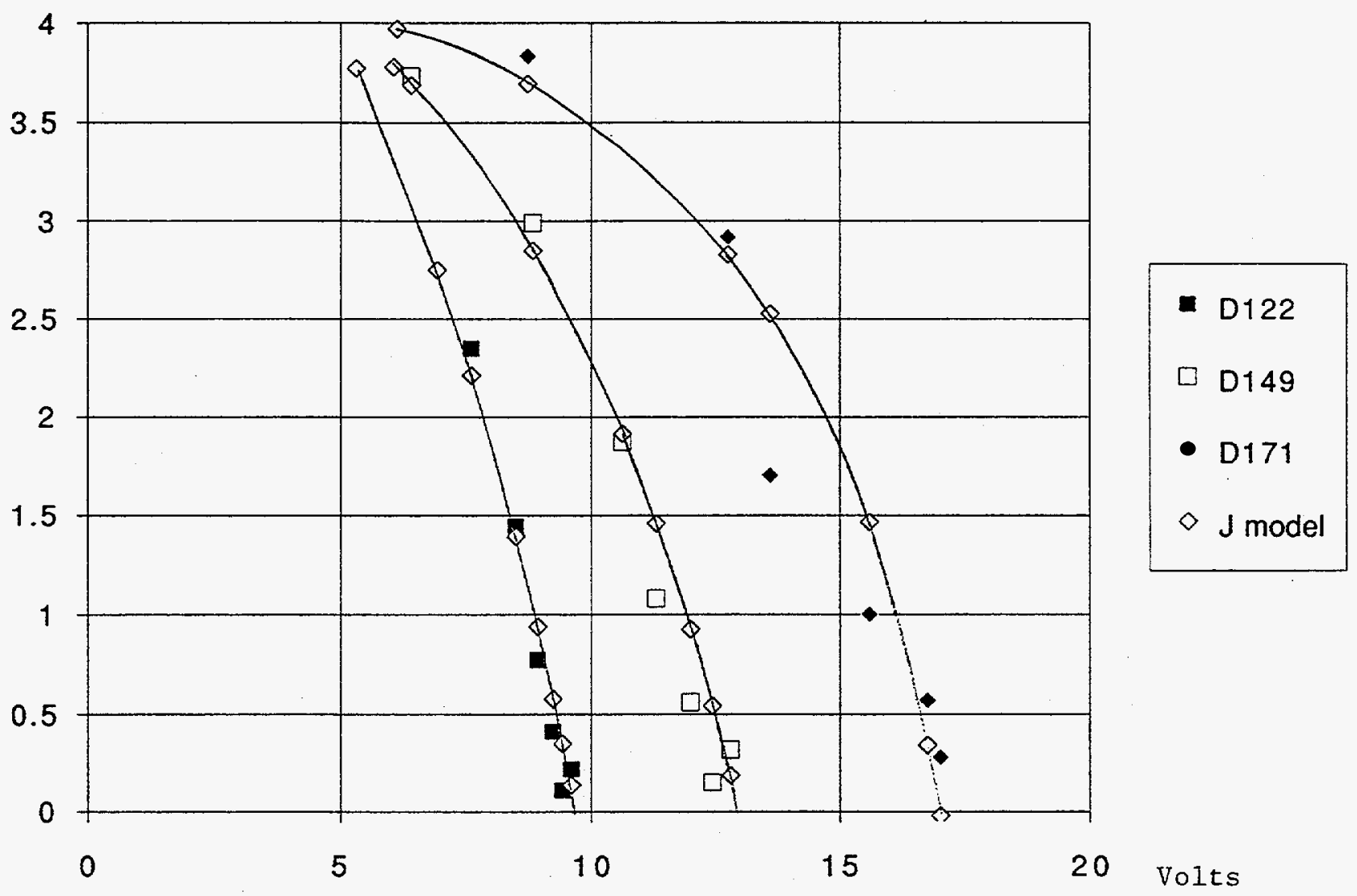

Fig. 2b. Calculated one parameter $\left(j_{i b} / j_{i p}\right)$ fits to the $J-V$ data plotted at sited: D122; D149; and D171. 


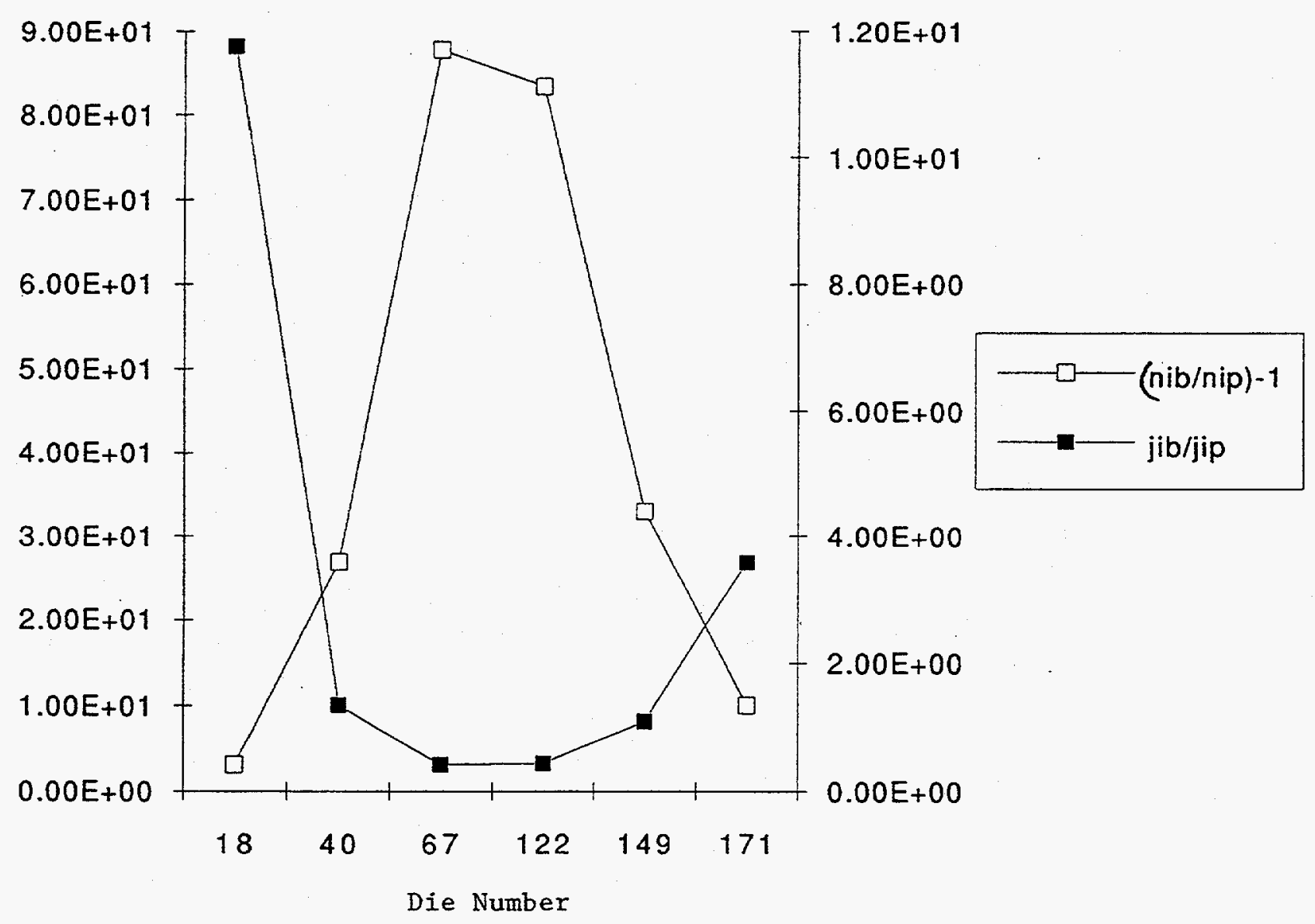

Fig. 3. Fitting parameter values used to match the $J-V$ curves: $j_{i b} / j_{i p}$ (right axis) and $n_{i p} / n_{i b}$ (left axis). 